\title{
THE CYLINDER PROBLEM IN VISCOELASTIC STRESS ANALYSIS*
}

\author{
BY \\ T. G. ROGERS** AND E. H. LEE \\ Stanford University
}

\begin{abstract}
A short review is presented of the cylinder problem as a vehicle for developments in the theory of linear viscoelastic stress analysis. This is followed by the solution of the problem of a compressible, hollow circular viscoelastic cylinder encased in and bonded to an elastic cylindrical shell. The analysis includes the effects of arbitrarily varying angular velocity and internal pressure, and the inner surface may ablate at an arbitrary rate. Material properties are incorporated in the form of numerical values of the relaxation modulus in shear, and the bulk modulus. Results are presented and comparison made with previous solutions which deal with more restricted situations.

1. Introduction. The determination of the stress distribution in a hollow cylinder subjected to internal pressure, and deforming under plane strain with axial motion prevented, has provided a convenient vehicle for several developments in viscoelastic theory. It is also of some technical interest, since the body may be considered to represent a cylindrical propellant grain in a solid fuel rocket. In this paper we shall consider the problem for a linear viscoelastic material on the basis of infinitesimal strain theory for which, as in classical elasticity, the boundary conditions are assumed satisfied on the undeformed boundary. A review of previous attempts to solve this problem is first presented.
\end{abstract}

In contrast with some examples discussed later, which include ablating surfaces, the case of a geometrically invariant viscoelastic body with boundary conditions at a surface point always of the same type (i.e. either stress or displacement history known) is formally solved in a straight forward manner by the application of the Laplace transform, which determines the associated elastic problem [1]†. This is particularly simple for the circular cylinder with a concentric circular cavity, since in this case the stress distribution for the associated elastic problem is given by the Lamé solution [2]:

$$
\begin{aligned}
& \sigma_{r}^{*}(r, p)=A^{*}(p)-\frac{B^{*}(p)}{r^{2}}, \\
& \sigma_{\theta}^{*}(r, p)=A^{*}(p)+\frac{B^{*}(p)}{r^{2}},
\end{aligned}
$$

where the star denotes the Laplace transform with respect to the time variable, $p$ is the transform parameter, $\sigma_{r}$ and $\sigma_{\theta}$ are the radial and circumferential stress components of the principal stress triad $\left(\sigma_{r}, \sigma_{\theta}, \sigma_{z}\right)$, and $A^{*}$ and $B^{*}$ are arbitrary functions of $p$ to be determined from the boundary conditions defined at the internal and external radii $r=a$ and $r=b$. If these boundary conditions are prescribed pressure histories, then

*Received July 31, 1963. Sponsored under Department of Defense Grant DA-ARO(D) 31-124-G-367 by the U. S. Army Research Office (Durham) to Stanford University.

**Now at the University of Nottingham, England.

$\nmid$ Numbers in square brackets refer to the bibliography at the end of the paper. 
Eq. (1) shows that $A^{*}$ and $B^{*}$ are independent of the associated elastic constants; hence inversion of Eqs. (1) and (2) demonstrates that, as in the elastic problem, the stress components $\sigma_{r}$ and $\sigma_{\theta}$ are independent of the material characteristics of the cylinder, and are therefore identical with the corresponding elastic solution at each instant. For these components we thus have an example of Alfrey's Theorem as generalized for compressible media [1].

If the outer boundary condition is that the cylinder is constrained in an elastic casing [2], then $A^{*}$ and $B^{*}$ will depend on associated elastic constants, and inversion of the transformed quantities will show that $\sigma_{r}(r, t)$ and $\sigma_{\theta}(r, t)$ will vary with time even for constant internal pressure. Thus the viscoelastic material behavior causes variation of the stress distribution for constant surface traction, comprising a more general type of solution than those satisfying Alfrey's Theorem.

When the inner surface is not only pressurized, but also is ablating, so that the surface is now defined as $r=a(t)$, the Laplace transform approach is no longer directly applicable [3]. Hence a different approach of direct integration of the equations of stress analysis was attempted. For quasi-static analysis, the equilibrium equations and the strain-displacement relations contain only space derivatives, while the constitutive equations contain only operators in the time variable. It is thus conceivable that integration of the former with respect to space variables, and of the latter with respect to time can be carried out independently. The case of the ablating cavity with prescribed inner pressure and external elastic support of the cylinder was first treated in [2], but could be solved only for the special case of an incompressible material exhibiting the Kelvin or Voigt type viscoelastic property in shear, corresponding to the model of a spring and dashpot in parallel. Incompressibility permits a simple analysis in terms of the radial displacement $u(r, t)=k(t) / r$, and satisfaction of the equation of equilibrium and the linear viscoelastic shear relation:

$$
P(\tau)=Q(\gamma / 2),
$$

where $P$ and $Q$ are linear operators in time, $\tau$ is the shear stress and $\gamma$ the shear strain, leads to the equation:

$$
P\left[\sigma_{r}(r, t)\right]=-\frac{Q[k(t)]}{r^{2}}-\frac{1}{b^{2}}\left(\frac{P}{C}-Q\right)[k(t)],
$$

where $C$ is a constant determined by the stiffness of the casing. The operator $P$ on the left hand side pertains to a particular material particle, and hence must be applied at constant $r . k(t)$ is to be determined in terms of the prescribed cavity pressure:

$$
p(t)=-\sigma_{r}[a(t), t] .
$$

If the operator $P$ is other than multiplication by a constant, the left hand side of (4) cannot be evaluated directly from (5), which applies for the variable radius $r=a(t)$, so that $k(t)$ cannot be determined from (4) without first eliminating the operator $P$. For Kelvin type behavior:

$$
P=1, \quad Q=H(\partial / \partial t)+J,
$$

where $H$ and $J$ are constants, the left hand side of (4) is equal to $-p(t)$ for $r=a(t)$ by (5) and (6), and (4) then constitutes a differential equation for $k(t)$ as detailed in [2]. Since only differential operator viscoelastic laws were considered there, more general 
material behavior could not be introduced since the resulting $\partial / \partial t$ derivatives in $P$ could not be evaluated directly on the left hand side of (4).

Retaining differential operator laws, attempts were made in $[4,5]$ to integrate with respect to time the equations corresponding to (4) when the material was considered to be elastically compressible and centrifugal forces associated with spin around the axis were added to the internal pressure loading. Both pressure and spin were considered to be generated discontinuously at $t=0$, and the problem of evaluating initial conditions for the resulting differential equations proved to be a non-trivial problem. With high order differential operators, high order initial stress derivatives are needed, and these are influenced by the viscoelastic material characteristics and the initial motion of the ablating cavity given by $a(t)$. In [4] delta functions and their derivatives were introduced to enable zero initial conditions to be assumed at $t=0^{-}$, before load application. In [5] initial conditions at $t=0^{+}$, immediately after load application, were evaluated separately. Both methods proved to be cumbersome, and only solutions involving the simpler outer boundary condition of prescribed external pressure were developed.

Although the integral operator form of (3), with

$$
P=1, \quad Q=2 \int_{0^{-}}^{t} G(t-\tau) \frac{\partial}{\partial \tau} d \tau,
$$

represents the most general formulation of linear viscoelastic behavior, Shinozuka [6] was the first to apply it to the problem of the ablating cylinder enclosed in an elastic casing. The representation (7) permits (4) to be utilized for general linear material behavior, by appropriate choice of the relaxation modulus $G(t)$. The application in [6] is, however, restricted to the case of standard linear solid viscoelastic behavior for which $G^{\prime}(t)$ is a single exponential function; Shinozuka then transformed the equivalent of (4) into a first-order differential equation which can be solved by the integrating factor technique. More elaborate mechanical model representation, however, could not be handled in this way.

The success of numerical solution of the Volterra type integral equations in viscoelastic stress analysis problems of this nature [7] suggests application of this technique to (4) and (7) for actual measured material relaxation moduli $G(t)$.

It will be shown below (Section 2) that the problem for the more general case of an elastically compressible viscoelastic hollow cylinder supported by an elastic casing, with centrifugal body forces associated with spin and ablating at an arbitrary rate, can be analyzed in integral equation form suitable for numerical evaluation. The analysis will not now, however, reduce to the single equation of the form of (4), but to a pair of interrelated integral equations of convolution type, which are solved (Section 3) by finite difference techniques and with the aid of a high-speed digital computer. Particular cases are solved to illustrate features of the solution, and for comparison with the more restricted solutions in the literature cited above; the results are discussed in Section 4.

2. Development of the theory. For an annular cylinder (Fig. 1) with axial symmetry and loading limited to internal pressure and centrifugal body force due to spin, symmtery determines the cylindrical stress components $\sigma_{r}, \sigma_{\theta}$ and $\sigma_{z}$ to be principal stresses throughout the loading history. For varying angular velocity, the required torque would demand shear on coaxial cylindrical surfaces, but for a linear problem this can be treated independently and superposed on the present solution, and it is not dealt with below. Moreover, such shear only occurs during the spin-up period for a cylinder accelerated to a 


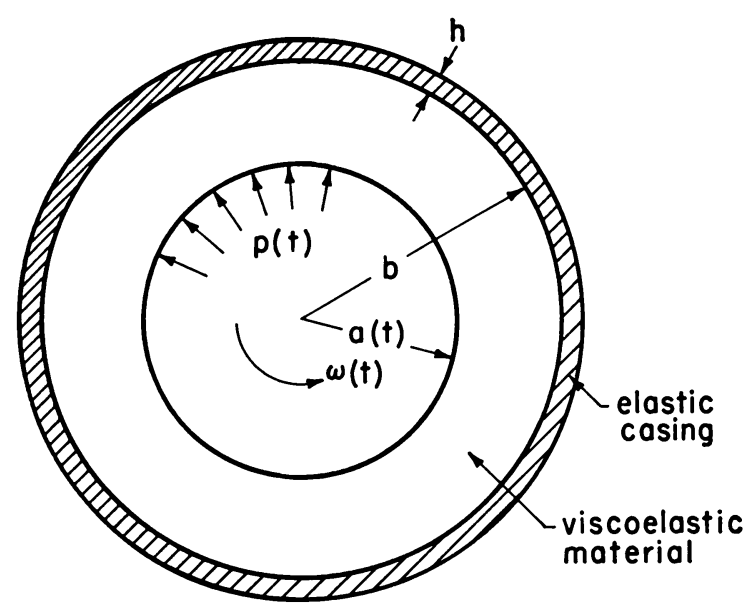

FIg. 1. Cross-section of the reinforced viscoelastic cylinder.

constant angular velocity. The equations of equilibrium thus reduce to

$$
r \frac{\partial \sigma_{r}}{\partial r}+\sigma_{r}-\sigma_{\theta}+\rho \omega^{2} r^{2}=0,
$$

where $\rho$ is the mass density of the viscoelastic material and $\omega=\omega(t)$ is the angular velocity of rotation. If the corresponding principal strains are $\epsilon_{r}, \epsilon_{\theta}$ and $\epsilon_{z}$, and $u$ is the radial displacement, then the strain-displacements relations and the condition of plane strain result in

$$
\epsilon_{r}=\frac{\partial u}{\partial r}, \quad \epsilon_{\theta}=\frac{u}{r}, \quad \epsilon_{z}=0 .
$$

Since, for mathematical convenience and lack of contrary experimental evidence for most viscoelastic materials, the material is assumed to be elastic in dilation with bulk modulus $k$, and viscoelastic in shear with the body undisturbed prior to zero time, the constitutive equations reduce to

$$
\begin{gathered}
\sigma_{r}+\sigma_{\theta}+\sigma_{z}=3 k\left(\epsilon_{r}+\epsilon_{\theta}+\epsilon_{z}\right)=3 k\left(\frac{\partial u}{\partial r}+\frac{u}{r}\right) \\
\sigma_{\theta}-\sigma_{r}=2 \int_{0^{-}}^{t} G(t-\tau) \frac{\partial}{\partial \tau}\left(\epsilon_{\theta}-\epsilon_{r}\right) d \tau=2 \int_{0^{-}}^{t} G(t-\tau) \frac{\partial}{\partial \tau}\left(\frac{u}{r}-\frac{\partial u}{\partial r}\right) d \tau, \\
\sigma_{\theta}-\sigma_{z}=2 \int_{0^{-}}^{t} G(t-\tau) \frac{\partial}{\partial \tau}\left(\epsilon_{\theta}-\epsilon_{z}\right) d z=2 \int_{0^{-}}^{t} G(t-\tau) \frac{\partial}{\partial \tau}\left(\frac{u}{r}\right) d \tau .
\end{gathered}
$$

The boundary conditions to be satisfied are those of prescribed internal pressure $p(t)$

$$
\sigma_{r}[a(t), t]=-p(t),
$$

and bonding at the elastic casing

$$
\sigma_{r}[b, t]=-B \epsilon_{\theta}(b, t) .
$$


In (14), $B$ is a constant determined by the properties of the elastic casing, assuming membrane theory, and is given by (refer [8])

$$
\frac{1}{B}=\left[1+\frac{b}{h}\left(1-\nu_{R}\right)\right] \frac{1+\nu_{R}}{E_{R}},
$$

where $h, E_{R}$ and $\nu_{R}$ are the thickness, Young's modulus and Poisson's ratio, respectively, of the reinforcing elastic material. Equation (14) neglects inertia forces in the ring; if these are significant, a term proportional to $\omega^{2}$ should be added to the right-hand side of (14), which adds a known function of time to the right-hand side of (19), without essential modification in the method of solution.

Equations (8), (10), (11) and (12) are four equations for the four unknowns $\sigma_{r}$, $\sigma_{\theta}, \sigma_{z}$ and, through $u, \epsilon_{\theta}$. The two boundary conditions, however, involve only two of them- $\sigma_{r}$ and $\epsilon_{\theta}$-and hence suggest the elimination of $\sigma_{\theta}$ and $\sigma_{z}$ as developed below. Eqs. (8), (9) and (11) may be combined to give

$$
r \frac{\partial \sigma_{r}}{\partial r}+\rho r^{2} \omega^{2}=-2 r \int_{0^{-}}^{t} G(t-\tau) \frac{\partial^{2} \epsilon_{\theta}}{\partial r \partial \tau} d \tau
$$

and hence, by integrating once with respect to $r$,

$$
\sigma_{r}=-\frac{1}{2} \rho r^{2} \omega^{2}-2 \int_{0^{-}}^{t} G(t-\tau) \frac{\partial \epsilon_{\theta}}{\partial \tau} d \tau+f(t),
$$

where $f(t)$ is an arbitrary function. Removing the discontinuity at $t=0$ and integrating by parts, we obtain

$$
\sigma_{r}(r, t)=f(t)-\frac{1}{2} \rho r^{2} \omega^{2}(t)-2 G(0) \epsilon_{\theta}(r, t)-2 \int_{0^{+}}^{t} \epsilon_{\theta}(r, \tau) G^{\prime}(t-\tau) d \tau,
$$

where the prime denotes differentiation with respect to the argument. Hence, using the boundary condition (14) we get a convolution-type integral equation of the second kind for $\sigma_{r}(b, t)$ :

$$
\sigma_{r}(b, t)+\frac{2 \mu}{E} \int_{0}^{t} G^{\prime}(t-\tau) \sigma_{r}(b, \tau) d \tau=\frac{\mu B}{E}\left[\frac{1}{2} \rho b^{2} \omega^{2}(t)-f(t)\right],
$$

where

$$
\mu=\frac{E}{2 G(0)-B}
$$

To obtain another integral equation for $\sigma_{r}(b, t)$ and $f(t)$ we eliminate first $\sigma_{z}$ from Eqs. (10) and (12), then $\sigma_{\theta}$ by using Eq. (11), and finally $u$ by (9) to end with

$$
3 \sigma_{r}=\frac{3 k}{r} \frac{\partial}{\partial r}\left(r^{2} \epsilon_{\theta}\right)+2 \int_{0^{-}}^{t} G(t-\tau) \frac{\partial}{\partial \tau}\left(\epsilon_{\theta}+2 r \frac{\partial \epsilon_{\theta}}{\partial r}\right) d \tau .
$$

The boundary condition involving $\epsilon_{\theta}$ has already been used, and the other contains only $\sigma_{r}$; hence the terms in $\epsilon_{\theta}$ in Eq. (21) are eliminated in favor of $\sigma_{r}$ and $f(t)$ by again using Eqs. (16) and (17). The $\epsilon_{\theta}$ term in the integrand is removed by subtracting Eq. (17) and twice Eq. (16) to give

$$
\Omega(r, t)=\frac{1}{2} f(t)-\frac{5}{4} \rho r^{2} \omega^{2}+\frac{3 k}{2 r} \frac{\partial}{\partial r}\left(r^{2} \epsilon_{\theta}\right),
$$


where for convenience we introduce

$$
\Omega(r, t)=2 \sigma_{r}+r \frac{\partial \sigma_{r}}{\partial r}=\frac{1}{r} \frac{\partial}{\partial r}\left(r^{2} \sigma_{r}\right) .
$$

The remaining term in $\epsilon_{\theta}$ is now removed from the analysis by adding twice Eq. (17) to Eq. (16) to give an equation

$$
\Omega(r, t)=2 f(t)-2 \rho r^{2} \omega^{2}(t)-\frac{2}{r} \int_{0^{-}}^{t} G(t-\tau) \frac{\partial^{2}}{\partial \tau \partial r}\left(r^{2} \epsilon_{\theta}\right) d \tau
$$

containing $\epsilon_{\theta}$ only in the form $\partial / \partial r\left(r^{2} \epsilon_{\theta}\right)$, which may now be simply eliminated by using Eq. (22) to give

$$
\begin{aligned}
\Omega(r, t)+\frac{4}{3 k} \int_{0^{-}}^{t} G(t-\tau) \frac{\partial \Omega(r, \tau)}{\partial \tau} d \tau & =2 f(t)-2 \rho r^{2} \omega^{2}(t) \\
+ & \frac{1}{3 k} \int_{0^{-}}^{t} G(t-\tau) \frac{\partial}{\partial \tau}\left[2 f(\tau)-5 \rho r^{2} \omega^{2}(\tau)\right] d \tau .
\end{aligned}
$$

As discussed in the Introduction, it is difficult to introduce the boundary condition at the ablating surface unless the time operator on the stress is removed. Hence we introduce the auxiliary $R$-function defined by

$$
R(t)+\frac{4}{3 k} \int_{0^{-}}^{t} G(t-\tau) \frac{\partial R(\tau)}{\partial \tau} d \tau=2 G(t)
$$

so that, using the associative property of convolutions, Eq. (25) may be rewritten as

$$
\Omega(r, t)=2 f(t)-2 \rho r^{2} \omega^{2}(t)+\frac{1}{k} \int_{0^{-}}^{t} R(t-\tau) \frac{\partial}{\partial \tau}\left[\frac{1}{2} \rho r^{2} \omega^{2}(\tau)-f(\tau)\right] d \tau .
$$

Removing the discontinuity at $t=0$, and integrating by parts, gives

$$
\begin{aligned}
\Omega(r, t)=\left[2-\frac{R(0)}{k}\right] f(t) & -\rho r^{2} \omega^{2}(t)\left[2-\frac{R(0)}{2 k}\right] \\
& -\frac{1}{k} \int_{0^{+}}^{t} R^{\prime}(t-\tau) f(\tau) d \tau+\frac{\rho r^{2}}{2 k} \int_{0^{+}}^{t} R^{\prime}(t-\tau) \omega^{2}(\tau) d \tau .
\end{aligned}
$$

Recalling (23), integrating with respect to $r$, and using the boundary condition (13) gives

$$
\begin{aligned}
& r^{2} \sigma_{r}(r, t)=\frac{1}{2}\left[r^{2}-a^{2}(t)\right]\left[\left\{2-\frac{R(0)}{k}\right\} f(t)-\frac{1}{k} \int_{0}^{t} R^{\prime}(t-\tau) f(\tau) d \tau\right] \\
& -\frac{\rho}{4}\left[r^{4}-a^{4}(t)\right]\left[\left\{2-\frac{R(0)}{2 k}\right\} \omega^{2}(t)-\frac{1}{2 k} \int_{0}^{t} R^{\prime}(t-\tau) \omega^{2}(\tau) d \tau\right]-a^{2}(t) p(t) .
\end{aligned}
$$

In particular this is true at $r=b$, and hence

$$
\begin{aligned}
& b^{2} \sigma_{r}(b, t)=\frac{1}{2}\left[b^{2}-a^{2}(t)\right]\left[\left\{2-\frac{R(0)}{k}\right\} f(t)-\frac{1}{k} \int_{0}^{t} R^{\prime}(t-\tau) f(\tau) d \tau\right] \\
& -\frac{\rho}{4}\left[b^{4}-a^{4}(t)\right]\left[\left\{2-\frac{R(0)}{2 k}\right\} \omega^{2}(t)-\frac{1}{2 k} \int_{0}^{t} R^{\prime}(t-\tau) \omega^{2}(\tau) d \tau\right]-a^{2}(t) p(t) .
\end{aligned}
$$

This equation is a convolution type integral equation for $f(t)$, and together with Eq. (19) forms a pair of interrelated integral equations in the unknown functions $f(t)$ and $\sigma_{r}(b, t)$. 
They may be solved numerically, as described in the next section, by simultaneous solution of their appropriate finite-difference forms. Once $f(t)$ is determined, $\sigma_{r}(r, t)$ may be obtained from Eq. (27), and hence $\sigma_{\theta}(r, t)$ is determined from Eq. (26) and

$$
\sigma_{\theta}(r, t)=\Omega(r, t)-\sigma_{r}(r, t)+\rho r^{2} \omega^{2}(t) .
$$

Finally $\epsilon_{\theta}(r, t)$ and $u(r, t)$ may be obtained by solving the integral equation (18) knowing $\sigma_{r}(r, t)$ and $f(t)$, or by using the straightforward integral representation obtained by inverting Eq. (17) to give

$$
\epsilon_{\theta}(r, t)=\frac{1}{2} \int_{0^{-}}^{t} J(t-\tau) \frac{\partial}{\partial \tau}\left[f(\tau)-\sigma_{r}(r, \tau)-\frac{1}{2} \rho r^{2} \omega^{2}(\tau)\right] d \tau .
$$

Knowing $f(t)$ and $\sigma(r, t)$, this integral can be computed by straightforward quadratures, after first removing the discontinuity at $t=0$. The creep function $J(t)$ may either be itself a measured material characteristic, or be determined numerically from the relaxation modulus $G(t)$ as in [9].

The restricted situation of incompressible material behavior and no spin represented by Eq. (4) can be reproduced from (19) and (28) by substituting $\omega=0, k=\infty, \sigma_{r}(b, t)=$ $-B k(t) / b^{2}$ and eliminating $f(t)$.

3. Method of solution. We first introduce the following dimensionless quantities:

$$
\begin{gathered}
t^{0}=t / t_{0}, \quad \sigma^{0}\left(t^{0}\right)=\frac{\sigma_{r}(b, t)}{E}, \quad f^{0}\left(t^{0}\right)=\frac{f(t)}{E}, \quad B^{0}=B / E, \quad G^{0}\left(t^{0}\right)=\frac{G(t)}{E}, \\
R^{0}\left(t^{0}\right)=\frac{R(t)}{E}, \quad \omega^{0}\left(t^{0}\right)=\frac{\rho b^{2} \omega^{2}(t)}{4 E}, \quad a^{0}\left(t^{0}\right)=\frac{a^{2}(t)}{b^{2}}, \quad p^{0}\left(t^{0}\right)=\frac{p(t)}{E},
\end{gathered}
$$

where $t_{0}$ is the total time of ablation, and $E$ is the initial value of the Young's modulus of the viscoelastic material. Eqs. (19) and (28) may now be rewritten in their dimensionless forms

$$
\begin{gathered}
\sigma^{0}\left(t^{0}\right)=2 \mu \int_{0}^{t^{0}} \sigma^{0}(\tau) \frac{1}{\partial \tau} G^{0}\left(t^{0}-\tau\right) d \tau+\mu B^{0}\left[2 \omega^{0}\left(t^{0}\right)-f^{0}\left(t^{0}\right)\right], \\
\sigma^{0}\left(t^{0}\right)=2 L\left(t^{0}\right) \int_{0}^{t^{\circ}} f^{0}(\tau) \frac{\partial}{\partial \tau} R^{0}\left(t^{0}-\tau\right) d \tau+H\left(t^{0}\right)+I\left(t^{0}\right) f^{0}\left(t^{0}\right),
\end{gathered}
$$

where

$$
\begin{aligned}
& L\left(t^{0}\right)=\frac{3}{4}\left(1-2 \nu_{0}\right)\left[1-a^{0}\left(t^{0}\right)\right], \\
& H\left(t^{0}\right)=-\left[1-\left\{a^{0}\left(t^{0}\right)\right\}^{2}\right]\left[\frac{3-2 \nu_{0}}{2\left(1-\nu_{0}\right)} \omega^{0}\left(t^{0}\right)+\frac{3\left(1-2 \nu_{0}\right)}{2} \int_{0}^{t^{\circ}} \omega^{0}(\tau) \frac{\partial}{\partial \tau} R^{0}\left(t^{0}-\tau\right) d \tau\right]-a^{0}\left(t^{0}\right) p^{0}\left(t^{0}\right), \\
& I\left(t^{0}\right)=\frac{1}{2\left(1-\nu_{0}\right)}\left[1-a^{0}\left(t^{0}\right)\right],
\end{aligned}
$$

with $\nu_{0}$ representing the initial Poisson's ratio of the viscoelastic material.

For convenience the superscript denoting a dimensionless variable is omitted in the sequel. As in [7], we now replace the integrals by their finite-sum approximations e.g.

$$
2 \int_{t_{1}=0}^{t_{n}=t} \sigma(\tau) \frac{\partial}{\partial \tau} G\left(t_{n}-\tau\right) d \tau \sim \sum_{k=1}^{n-1}\left[\sigma\left(t_{k}\right)+\sigma\left(t_{k+1}\right)\right]\left[G\left(t_{n}-t_{k+1}\right)-G\left(t_{n}-t_{k}\right)\right] .
$$


Hence now Eqs. (30) and (31) can be rewritten as

$$
\begin{gathered}
\sigma_{n}=\mu \sum_{k=1}^{n-1}\left(\sigma_{k+1}+\sigma_{k}\right)\left(G_{k+1}-G_{k}\right)+2 \mu B \omega_{n}-\mu B f_{n}, \\
\sigma_{n}=L_{n} \sum_{k=1}^{n-1}\left(f_{k+1}+f_{k}\right)\left(R_{k+1}-R_{k}\right)+H_{n}+I_{n} f_{n},
\end{gathered}
$$

where $G_{k}, R_{k}$ represent $G\left(t_{n}-t_{k}\right), R\left(t_{n}-t_{k}\right)$ respectively and elsewhere the quantity with suffix $k$ (for example) denotes the value of the function at time $t_{k}$. These two equations are now in the form of two simple simultaneous algebraic equations for $\sigma_{n}$ and $f_{n}$, and their solution is

$$
\begin{gathered}
f_{n}=\frac{\alpha_{n} \delta_{n}+\gamma_{n}}{B-\alpha_{n} \beta_{n}}, \\
\sigma_{n}=\frac{B \delta_{n}+\beta_{n} \gamma_{n}}{B-\alpha_{n} \beta_{n}}=\delta_{n}+\beta_{n} f_{n},
\end{gathered}
$$

where$$
\alpha_{n}=G_{n}-G_{n-1}-1 / \mu,
$$$$
\beta_{n}=I_{n}+L_{n}\left(R_{n}-R_{n-1}\right) \text {, }
$$$$
\gamma_{n}=\sigma_{n-1}\left(G_{n}-G_{n-1}\right)+2 B \omega_{n}+\sum_{k=1}^{n-2}\left(\sigma_{k+1}+\sigma_{k}\right)\left(G_{k+1}-G_{k}\right) \text {, }
$$$$
\delta_{n}=H_{n}+L_{n}\left[f_{n-1}\left(R_{n}-R_{n-1}\right)+\sum_{k=1}^{n-2}\left(f_{k+1}+f_{k}\right)\left(R_{k+1}-R_{k}\right)\right] .
$$

Equations (32)-(34) determine $f_{n}$ and $\sigma_{n}$ in terms of already determined quantities by taking successively $n=2,3 \cdots$ with the initial solution $(n=1)$ given by Eqs. (30) and (31) as

$$
f_{1}=\frac{2 \mu B \omega_{1}-H_{1}}{I_{1}+\mu B}, \quad \sigma_{1}=\mu B\left(2 \omega_{1}-f_{1}\right)
$$

The elastic solution is simply given by replacing $G(t)$ and $R(t)$ by appropriate constant values such as $G(0)$ and $R(0)$ respectively, which is equivalent to putting

$$
G_{k}=G_{n}, \quad R_{k}=R_{n}, \quad k=1,2 \cdots, n .
$$

The various numerical procedures described above were programmed for computation in the computer language BALGOL, and processed on the IBM 7090 of the Stanford University Computing Center. These facilities were in part made available under the NSF Grant NSF-GP948.

4. Results and discussion. The primary purpose of our presenting numerical solutions is to demonstrate the ease and accuracy of the method described above. In addition, however, it is possible to arrange the program of work in such a way that the effects and importance of various assumptions (for example, incompressibility) made in previous treatments of the cylinder problem may be assessed independently of one another.

First, the accuracy of the finite-sum approximation is assessed by using the numerical method to compute the stress distributions for the special case of a non-rotating incompressible cylinder, with standard linear solid behavior in shear given by

$$
G(t)=\frac{1}{3}\left[\alpha+(1-\alpha) e^{-\lambda t}\right]
$$


and subjected to an internal pressure

$$
p(t)=N t e^{-\alpha \lambda t}
$$

with the inner surface ablating according to

$$
a(t)=\frac{a_{0}}{\left[1-\left(1-a_{0}\right) t\right]},
$$

where $a_{0} \equiv a(0)$. In these relations, $\alpha, \lambda$ and $N$ are positive constants. The results may be compared with the analytical solution (derived in the Appendix) obtained by Shinozuka's method. As stated in the Introduction, this case is the most general to have been considered in the literature to date. For the particular case of

$$
\alpha=\frac{1}{2}, \quad \lambda=5, \quad a_{0}=\frac{4}{9}, \quad B=\frac{1}{3},
$$

the error in the numerical solution with fifty time steps $(\Delta t=0.02)$ is less than $0.05 \%$ at all_times and throughout the cylinder and is therefore insufficient to show in Fig. 2,

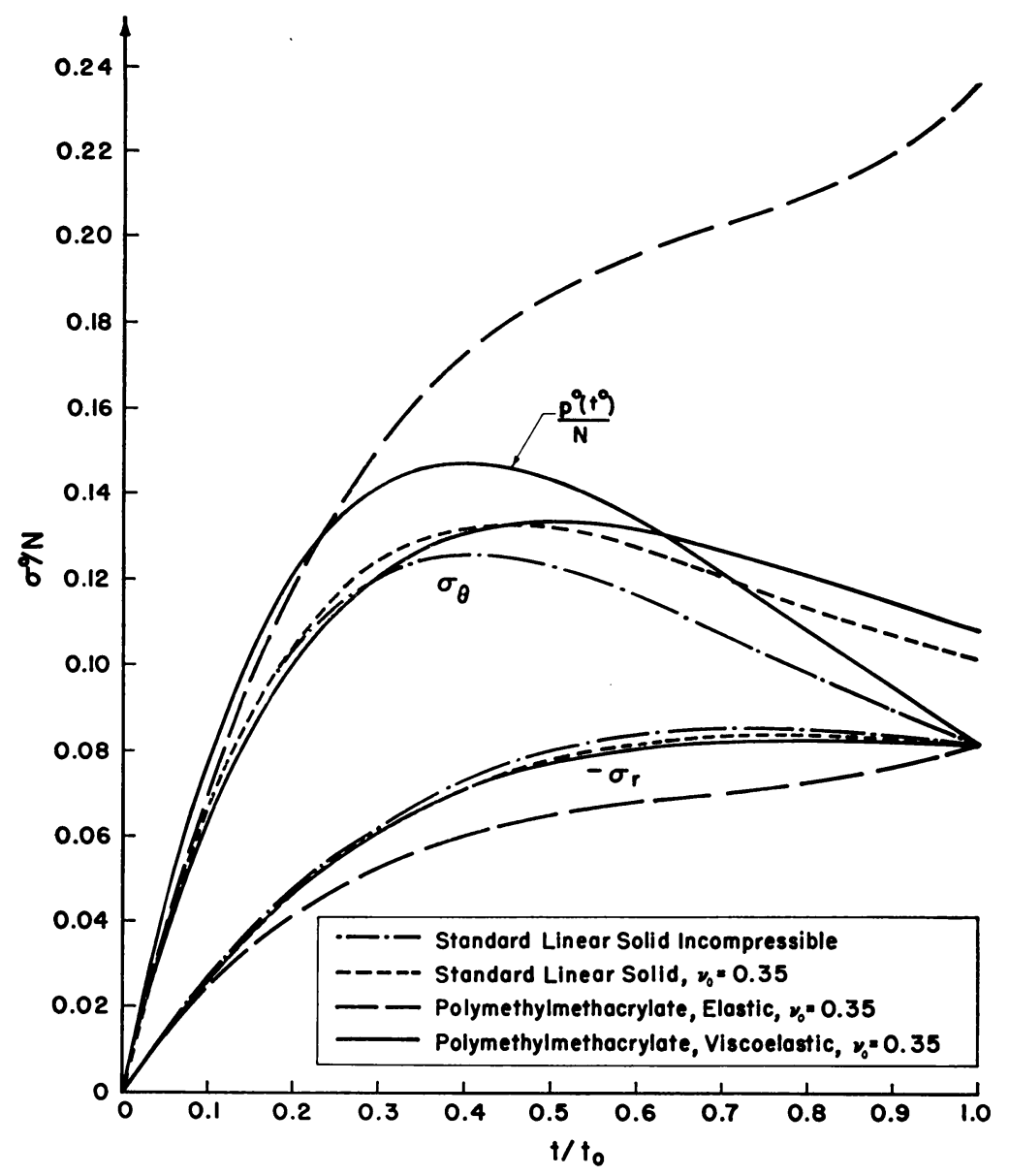

FIG. 2. Stress history adjacent to the elastic casing $(r=b)$. Effect of compressibility and model-fitting. 
which depicts the stress histories at the interface $(r=b)$ adjacent to the elastic casing. It appears from the figure that the radial and circumferential stresses are equal and of opposite sign at the moment of complete ablation; in fact this is not so, the difference (refer Appendix) being $2 f(1) / N$ which in the particular case computed is only 0.00037 , too small to be discerned in Fig. 2.

Figure 2, also shows the effect of compressibility which is now introduced by taking $\nu_{0}=0.35$. The radial stress distribution is hardly affected, but the circumferential stress distribution shows a marked change, increasing by up to $24 \%$ in this case.

One of the main advantages of the solution presented in this paper is demonstrated by next incorporating actual experimentally measured relaxation functions in shear, $G(t)$, without additional difficulty. For the viscoelastic material under consideration, polymethylmethacrylate, the experimental measurements of only the relaxation modulus in extension, $E(t)$, are available [10], but $G(t)$ and $R(t)$ are easily computed by the method described in [7]. $R(t)$ is given in [7]; $G(t)$ is shown in Figs. 3 and 4. Also shown

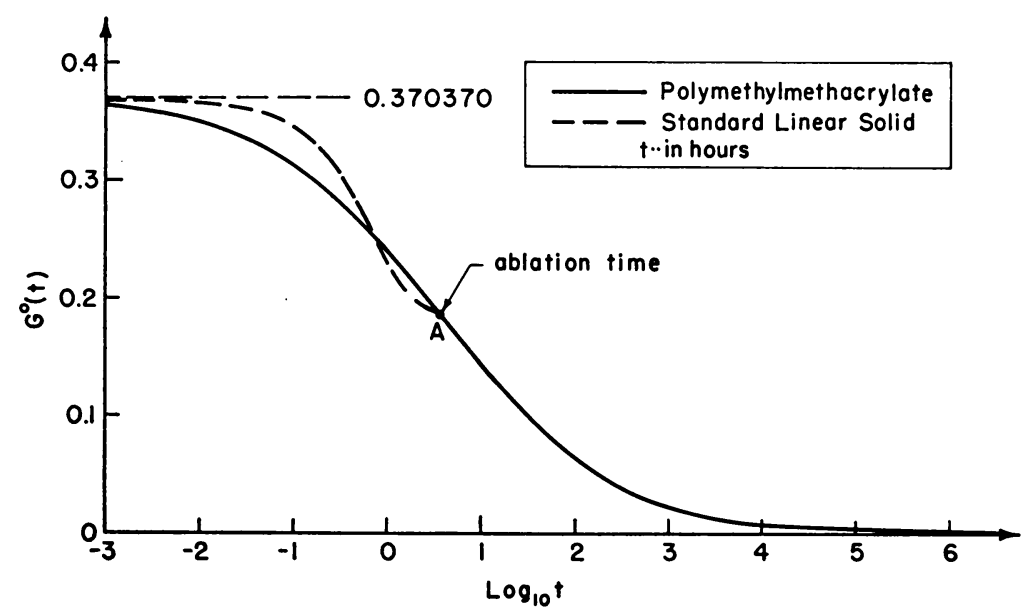

Fig. 3. Relaxation modulus in shear for polymethyl methacrylate at $80^{\circ} \mathrm{C}$ and fitted model (standard linear solid).

on these figures is the relaxation function $G(t)$ of the standard linear solid model chosen to fit the experimental curves at zero time, $t=3.64 \mathrm{hrs}$. and at an intermediate time. As seen in Figs. 3 and 4, this standard linear solid relaxation modulus provides an approximation to the viscoelastic characteristics of polymethylmethacrylate for time up to about $5 \mathrm{hrs}$., but differs markedly at long times. It was used in the stress analysis problem for an ablation time of $3.64 \mathrm{hrs}$., and the results are shown in Fig. 2 for comparison with those based on the measured modulus function. The model approximation is seen to provide an accurate analysis for the radial stresses, with an error up to $6 \%$ for the circumferential stresses. This satisfactory approximation is associated with the smooth loading function assumed, and the corresponding relatively narrow frequency spectrum needed to represent it. For a longer ablation time, different model constants would be needed to provide satisfactory accuracy over the different time range, whereas for the integral operator approach no such change need be assessed. For a more complicated loading program, involving possibly reloading, which would require a wide frequency spectrum to represent it accurately, it would be impossible to achieve satis- 
factory accuracy throughout on the basis of a standard linear solid model. It is perhaps interesting to note that for a thermorheologically simple material [8], a particular model fit will be satisfactory for different time ranges at different temperatures, and that the present solution corresponds to much shorter ablation times at higher temperatures.

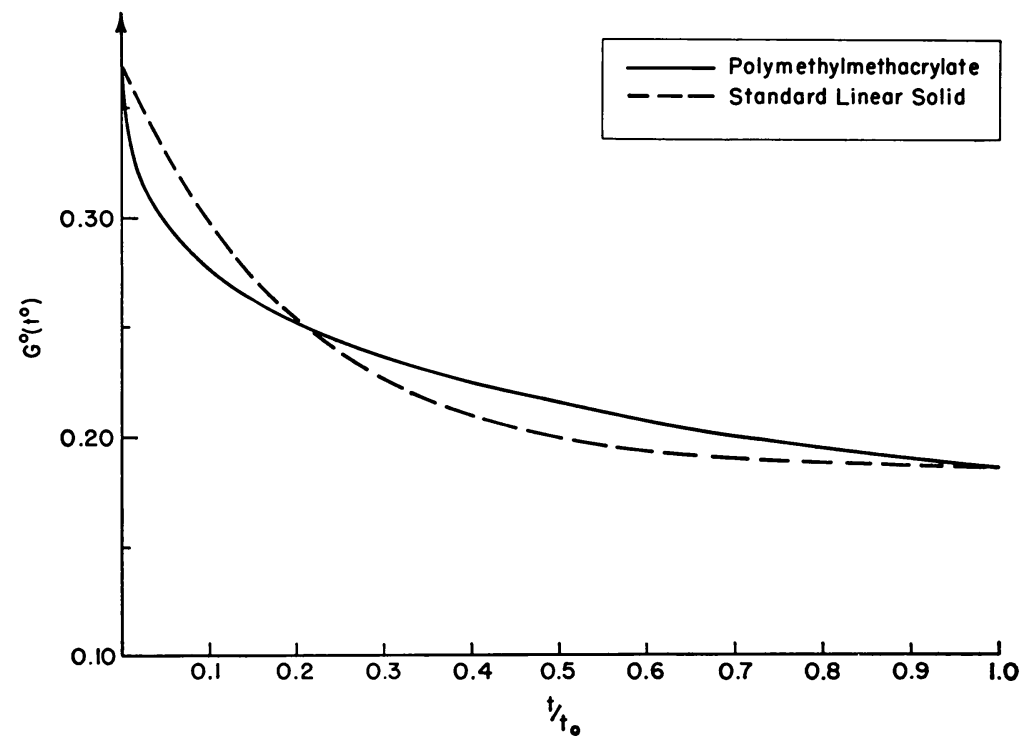

Fig. 4. Relaxation moduli in shear for polymethyl methacrylate and fitted model over time range of problem.

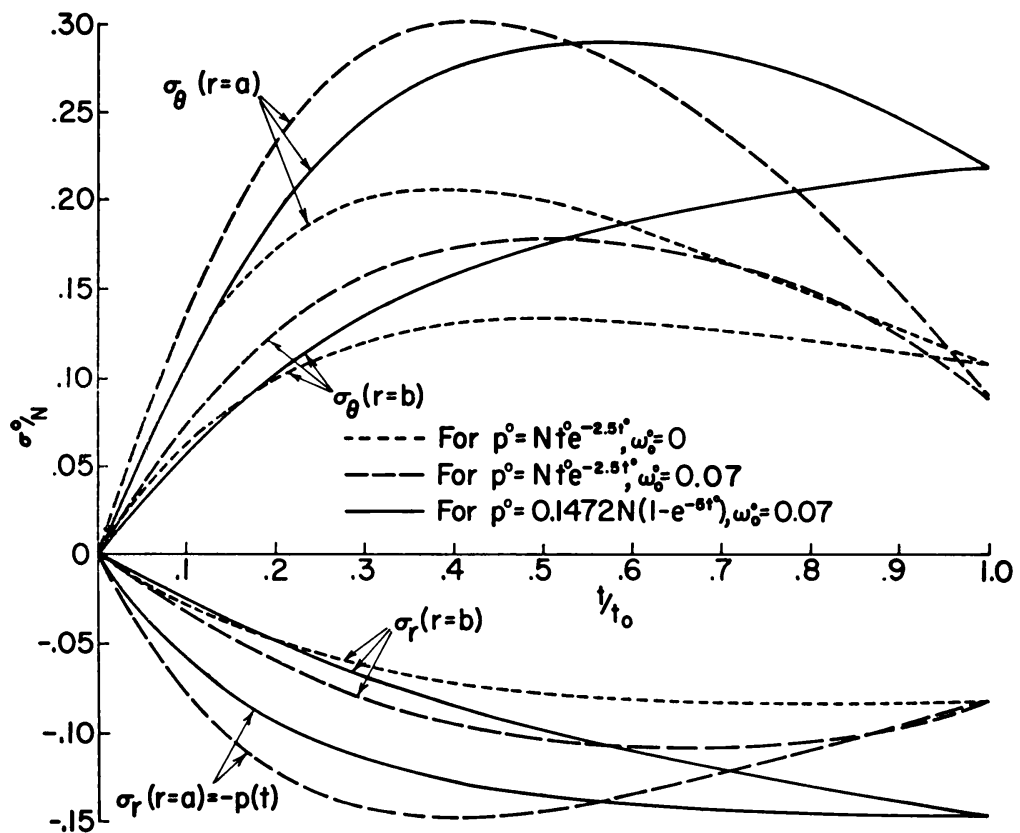

Fig. 5. Stress histories on the boundaries for different angular velocity and internal pressure variations. 


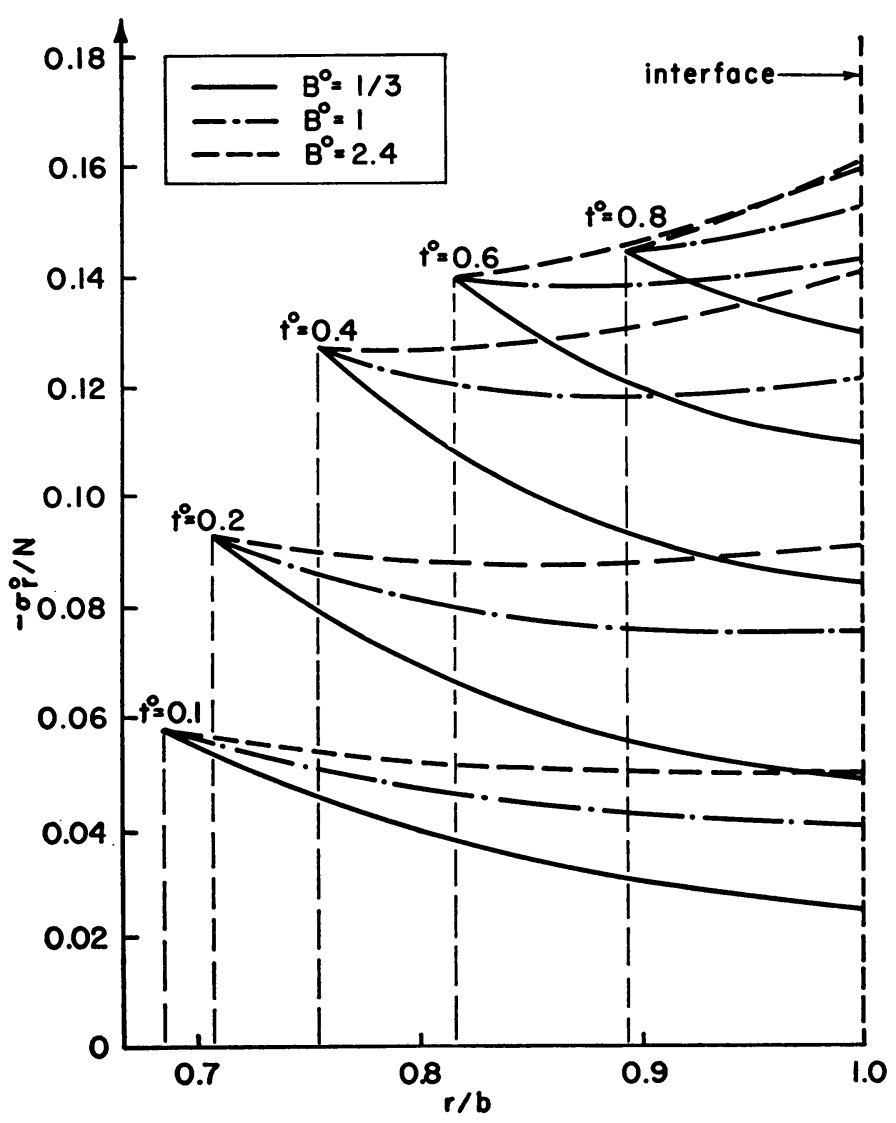

Fra. 6. Histories of radial stress distribution for different elastic casings.

The remaining curves in Fig. 2-the elastic solution with Young's modulus $E$ and Poisson's ratio $\nu_{0}$-demonstrate the marked influence of the viscoelastic properties of the material, and indicate their importance in the engineering design of equipment with viscoelastic components, such as a solid propellant rocket motor.

In dimensional quantities, the results indicate that if the internal pressure builds up to a maximum (occurring at $t=0.40$ ) of $p_{\max }=1000$ p.s.i., then the maximum circumferential stress $\sigma_{\theta}$ on the ablating inner surface occurs just before $t=0.40$ and equals about 2,800 p.s.i.; the maximum $\sigma_{\theta}$ on the interface $(r=b)$ occurs later at $t=0.51$ and is 1800 p.s.i. It is found that in this particular case the stress distributions at any time show a maximum magnitude at the inner surface and decrease monotonically to their minimum at the outer boundary.

Figure 5 shows the stress histories at the inner and outer boundaries caused by further applying different angular velocity and internal pressure histories defined respectively by

$$
\omega(t)=\omega_{0}\left(1-e^{-5 t}\right), \quad p(t)=p_{\infty}\left(1-e^{-5 t}\right) .
$$

These variations are chosen purely for demonstration purposes; quite arbitrary histories are as directly treated by the method. For purposes of comparison the value of the constant $p_{\infty}$ has been chosen to give the same maximum pressure $0.1472 E N$ as for the 


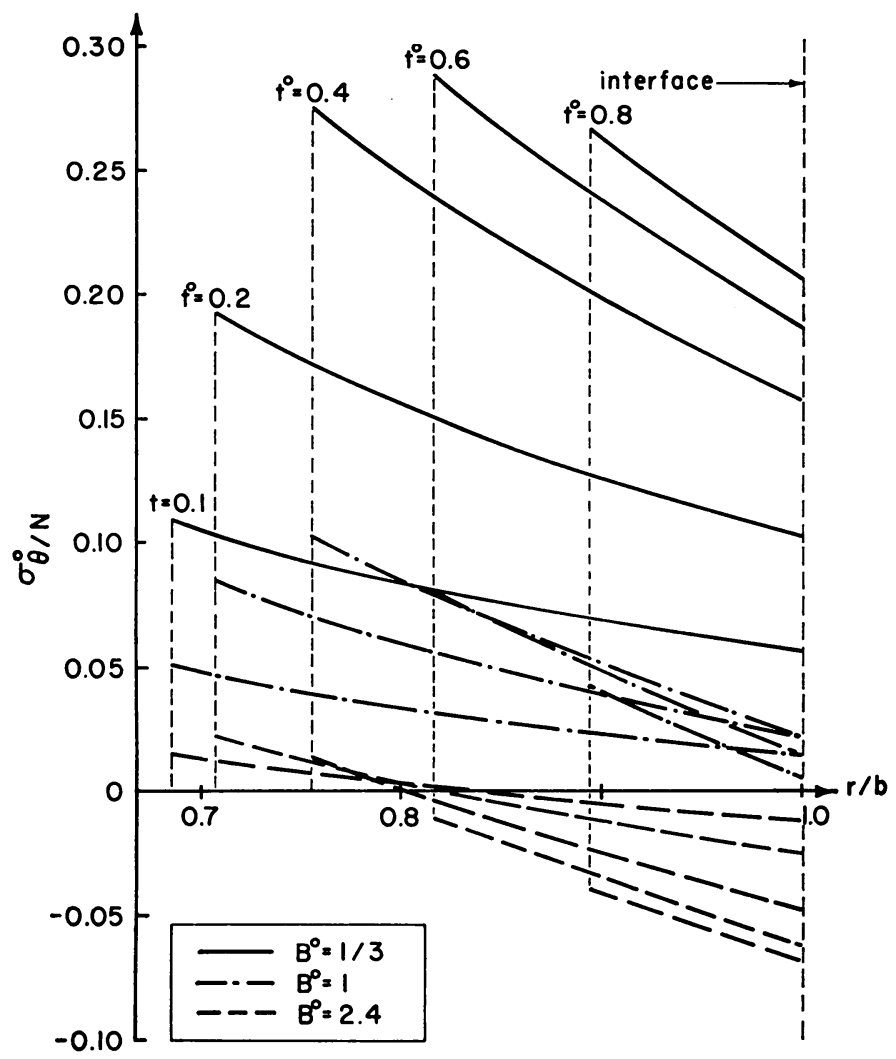

Fig. 7. Histories of circumferential stress distribution for different elastic casings.

previous pressure history (38). As might be expected, the introduction of spin and such a different pressure variation produces very different stress histories. Since the system is linear, the effect of a different $\omega_{0}$ may be readily calculated from the results given by the particular case of $\omega_{0}=0.07$, which is equivalent to a dimensional angular velocity of 86 revs-per sec. for

$p_{\max }=2000$ p.s.i., $\quad E=3.246 \times 10^{5}$ p.s.i., $\quad \nu_{0}=0.35 \rho=1 \mathrm{gm} / \mathrm{c} . \mathrm{c}, \quad b=30 \mathrm{cms}$.

Finally Figs. 6 and 7 demonstrate the effect of casings having different elastic properties. As in Shinozuka [6] the effect is dominant. The three cases considered:

$$
B=\frac{1}{3}, 1,2.4
$$

are chosen to indicate the effect of different elastic materials and different thickness ratios. The three values may be considered to correspond to aluminum with $b / h=100$, steel with the same thickness ratio, and steel with $b / h=40$, respectively. Not only are the magnitudes radically altered, but the signs of the circumferential stresses are changed. These results have obvious design implications. Compressive circumferential stresses are beneficial in preventing propagation of cracks; however, it should be noted that these stresses must be complemented by larger radial stresses, which at the interface $(r=b)$ would produce larger tensile circumferential stress in the casing. 


\section{APPENDIX}

5. An analytical solution for the incompressible case. For a non-rotating, incompressible cylinder, Eqs. (30) and (31) reduce to

$$
\begin{gathered}
\sigma(t)+2 \mu \int_{0}^{t} G^{\prime}(t-\tau) \sigma(\tau) d \tau=-\mu B f(t), \\
\sigma(t)=-a(t) p(t)+[1-a(t)] f(t) .
\end{gathered}
$$

We now follow Shinozuka's method, eliminating $f(t)$ from Eqs. (41) and (42) and using (37) to describe standard linear solid behavior in shear, to arrive at the Volterra integral equation of the second kind:

$$
\left[1+\frac{\mu B}{1-a(t)}\right] \sigma(t)-\frac{2}{3} \mu \lambda(1-\alpha) \int_{0}^{t} e^{-\lambda(t-\tau)} \sigma(\tau) d \tau=-\frac{\mu B a(t) p(t)}{1-a(t)}
$$

The solution of this equation is found to be

$$
\sigma(t)=\frac{e^{-\lambda t}}{F(t)}\left[H(t)+\exp \left\{\int_{0}^{t} \frac{d \tau}{F(\tau)}\right\} \int_{0}^{t} \frac{H(\tau)}{F(\tau)} \exp \left\{-\int_{0}^{\tau} \frac{d \zeta}{F(\zeta)}\right\} d \tau\right]
$$

where

$$
\begin{aligned}
& F(x)=\frac{1}{\lambda(1-\alpha)}[1+h(x)], \\
& H(x)=\frac{1}{\lambda(1-\alpha)} h(x) p(x) e^{\lambda x}, \\
& h(x)=\frac{3 B a(x)}{2[1-a(x)]} .
\end{aligned}
$$

The problem is now formally solved, but for comparison purposes an analytical solution requiring no numerical quadrature is needed. This is obtained by assuming (38) and (39), in which case Eq. (43) reduces to the simple form:

$$
\sigma(t)=\left\{\begin{array}{l}
-p(t)\left[1+\frac{1-t}{(\gamma-1) t}\left\{\left(1-\frac{t}{c}\right)^{\gamma-1}-1\right\}\right], \quad \gamma \neq 1 \\
-p(t)\left[1+\left(\frac{1}{t}-1\right) \ln \left(1-\frac{t}{c}\right)\right], \quad \gamma=1
\end{array}\right.
$$

where

$$
c=1+\frac{1}{\lambda(1-\alpha)}, \quad \gamma=\frac{h(0)}{c-1} .
$$

Using Eqs. (42) and (45) we also obtain

$$
f(t)= \begin{cases}-p(t)\left[1+\frac{1}{\gamma-1} \frac{1-\left(1-a_{0}\right) t}{t\left(1-a_{0}\right)}\left\{\left(1-\frac{t}{c}\right)^{\gamma-1}-1\right\}\right], & \gamma \neq 1, \\ -p(t)\left[1+\frac{1-\left(1-a_{0}\right) t}{t\left(1-a_{0}\right)} \ln \left(1-\frac{t}{c}\right)\right], & \gamma=1 .\end{cases}
$$


From Eqs. (25) and (29) we deduce

$$
\sigma_{r}(r, t)+\sigma_{\theta}(r, t)=\Omega(r, t)=2 f(t) .
$$

In particular, at $t=t_{0}$,

$$
\sigma_{r}(1,1)+\sigma_{\theta}(1,1)=\left\{\begin{array}{l}
-2 e^{-\lambda / 2}\left[1+\frac{a_{0}}{1-a_{0}}\left\{\left(1-\frac{1}{c}\right)^{\gamma-1}-1\right\}\right], \quad \gamma \neq 1, \\
-2 e^{-\lambda / 2}\left[1+\frac{a_{n}}{1-a_{0}} \ln \left(1-\frac{1}{c}\right)\right], \quad \gamma=1 .
\end{array}\right.
$$

Hence using (40) for the particular case computed in the paper, we obtain

$$
\sigma_{r}(1,1)+\sigma_{\theta}(1,1)=\mathrm{e}^{-5 / 2}(-1+0.8 \ln 3.5) \sim 0.00037 .
$$

\section{BibLIOGRAPHY}

1. E. H. Lee, Quart. Appl. Math. 13 (1955) 183-190

2. E. H. Lee, J. R. M. Radok and W. B. Woodward, Trans. Soc. Rheol. 3 (1959) 41-59

3. E. H. Lee and J. R. M. Radok, Proc. Ninth Int. Congs. Appl. Mech. 5 (1957) 321-329

4. A. H. Corneliussen and E. H. Lee, Proc. IUTAM Colloq. on Creep in Structures, Springer-Verlag, 1962 , pp. $1-20$

5. A. H. Corneliussen, E. F. Kamowitz, E. H. Lee and J. R. M. Radok, Trans. Soc. Rheol. 7 (1963)

6. M. Shinozuka, A. S. M. E. Paper No. 63-APMW-2, 1963

7. E. H. Lee and T. G. Rogers, J. Appl. Mech. 30 (1963) 127-133

8. L. W. Morland and E. H. Lee, Trans. Soc. Rheol. 4 (1960) 233-263

9. I. L. Hopkins and R. W. Hamming, J. Appl. Phys. 28 (1957) 906-909

10. J. Bischoff, E. Catsiff and A. V. Tobolsky, J. Am. Chem. Soc. 74 (1952) 3378-3381 